\title{
Article \\ The Capsid (ORF2) Protein of Hepatitis E Virus in Feces Is C-Terminally Truncated
}

\author{
Takashi Nishiyama ${ }^{1,2, *(\mathbb{D}, \text { Koji Umezawa }}{ }^{3}$, Kentaro Yamada ${ }^{1,4}$, Masaharu Takahashi ${ }^{1}$, Satoshi Kunita 5 , \\ Mulyanto ${ }^{6,7}$, Isao Kii ${ }^{2}$ and Hiroaki Okamoto ${ }^{1, *(\mathbb{D})}$
}

1 Division of Virology, Department of Infection and Immunity, Jichi Medical University School of Medicine, 3311-1 Yakushiji, Shimotsuke-shi 329-0498, Japan; kentaro-y@cc.miyazaki-u.ac.jp (K.Y.); mtaka84@jichi.ac.jp (M.T.)

2 Laboratory for Drug Target Research, Integrated Bioscience Division, Institute of Agriculture, Shinshu University, 8304 Minamiminowa, Kamiina 399-4598, Japan; ikii@shinshu-u.ac.jp

3 Department of Biomedical Engineering, Institute for Biomedical Sciences, Shinshu University, 8304 Minamiminowa, Kamiina 399-4598, Japan; koume@shinshu-u.ac.jp

4 Laboratory of Veterinary Public Health, Department of Veterinary Sciences, Faculty of Agriculture, University of Miyazaki, Miyazaki 889-2192, Japan

5 Center for Experimental Medicine, Jichi Medical University School of Medicine, 3311-1 Yakushiji, Shimotsuke-shi 329-0498, Japan; sakunita@jichi.ac.jp

6 West Nusa Tenggara Hepatitis Laboratory, Mataram 83231, Indonesia; mulyanto@unram.ac.id

7 Immunobiology Laboratory, Faculty of Medicine, University of Mataram, Mataram 83125, Indonesia

* Correspondence: tnishiyama@shinshu-u.ac.jp (T.N.); hokamoto@jichi.ac.jp (H.O.); Tel.: +81-265-77-1627 (T.N.); +81-285-58-7404 (H.O.)

check for updates

Citation: Nishiyama, T.; Umezawa,

K.; Yamada, K.; Takahashi, M.; Kunita, S.; Mulyanto; Kii, I.; Okamoto, H. The Capsid (ORF2) Protein of Hepatitis E Virus in Feces Is C-Terminally Truncated. Pathogens 2022, 11, 24. https://doi.org/ $10.3390 /$ pathogens 11010024

Academic Editors: Sven Pischke, Lin Wang and Jerome Gouttenoire

Received: 6 December 2021

Accepted: 24 December 2021

Published: 26 December 2021

Publisher's Note: MDPI stays neutral with regard to jurisdictional claims in published maps and institutional affiliations.

Copyright: (c) 2021 by the authors. Licensee MDPI, Basel, Switzerland. This article is an open access article distributed under the terms and conditions of the Creative Commons Attribution (CC BY) license (https:// creativecommons.org/licenses/by/ $4.0 /)$.

\begin{abstract}
The hepatitis E virus (HEV) is a causative agent of hepatitis E. HEV virions in circulating blood and culture media are quasi-enveloped, while those in feces are nonenveloped. The capsid (ORF2) protein associated with an enveloped HEV virion is reported to comprise the translation product of leucine 14/methionine 16 to 660 (C-terminal end). However, the nature of the ORF2 protein associated with fecal HEV remains unclear. In the present study, we compared the molecular size of the ORF2 protein among fecal HEV, cell-culture-generated HEV (HEVcc), and detergent-treated protease-digested HEVcc. The ORF2 proteins associated with fecal HEV were C-terminally truncated and showed the same size as those of the detergent-treated protease-digested HEVcc virions (60 kDa), in contrast to those of the HEVCc $(68 \mathrm{kDa})$. The structure prediction of the ORF2 protein (in line with previous studies) demonstrated that the C-terminal region ( 54 amino acids) of an ORF2 protein is in flux, suggesting that proteases target this region. The nonenveloped nondigested HEV structure prediction indicates that the $\mathrm{C}$-terminal region of the ORF2 protein moves to the surface of the virion and is unnecessary for HEV infection. Our findings clarify the maturation of nonenveloped HEV and will be useful for studies on the HEV lifecycle.
\end{abstract}

Keywords: hepatitis E virus; nonenveloped infectious virion; fecal sample; truncated ORF2 protein

\section{Introduction}

Hepatitis $\mathrm{E}$ is acute hepatitis, a generally self-limiting and rarely fatal disease, with a lethality of $0.5-3 \%$ among young adults; however, this rate reaches $20-30 \%$ in pregnant women [1,2]. This form of hepatitis is caused by hepatitis E virus (HEV) infection via the fecal-oral route, from polluted water in developing countries [1,2]. Furthermore, in developed countries, hepatitis $\mathrm{E}$ has been increasingly reported as a zoonotic food-borne transfusion-associated, or organ-transplantation-associated, disease [3,4], and chronic cases can occur in immunocompromised individuals, such as those receiving organ transplants, patients with hematological malignancy, and HIV-infected patients [5-7].

HEV belongs to the Hepeviridae family, which is classified into two genera: Orthohepevirus and Piscihepevirus [8]. The genus, Orthohepevirus, is divided into four species (A-D). The Orthohepevirus A species includes eight different HEV genotypes (1-8). Genotypes 
1-4 and 7 are known to infect humans [9], while genotypes 5 and 8 are reported to infect monkeys and are suggested to carry a risk for zoonotic infection $[10,11]$. Apart from the Orthohepevirus $A$ species, recent studies have documented zoonotic infections of humans with rat HEV, belonging to the Orthohepevirus $C$ species $[12,13]$.

HEV has an approximately 7.2-kilobase $(\mathrm{kb})$ single-stranded positive-sense RNA genome. This viral genome encodes three proteins: a nonstructural polyprotein required for RNA replication (ORF1); a capsid protein of the HEV virion (ORF2); and a small multifunctional protein with key functions in particle assembly and release (ORF3) [14,15]. The ORF2 protein contains three distinct domains: S (shell, amino acids [aa] 118-313); M (middle, aa 314-453); and P (protruding, aa 455-end) [16]. The $S$ domain is composed of jelly roll-like $\beta$-sheets; the $\mathrm{M}$ domain is tightly linked to the $\mathrm{S}$ domain and is located at the surface; and the $P$ domain dimerizes, forming protruding spikes [17-19]. The ORF2 protein is also secreted as dimerized and glycosylated protein [20]. The ORF3 protein is localized at the lipid membrane of the enveloped virion [21]. Both the ORF2 and ORF3 proteins are translated from a bicistronic subgenomic RNA, approximately $2.2 \mathrm{~kb}$ in length [22].

$\mathrm{HEV}$ is secreted into the culture media and the circulating blood as an enveloped HEV with a host-derived lipid membrane and ORF3 protein [23], but it is shed into feces as a nonenveloped HEV, without the lipid coat or ORF3 protein [21]; thus, it has been declared a "quasi-enveloped" virus [24]. Takahashi et al. [25] reported that detergent- and protease-treated enveloped HEV lost the ORF3 protein and lipid membrane and displayed a buoyant density of $1.27 \mathrm{~g} / \mathrm{mL}$, similar to fecal nonenveloped HEV, suggesting that the treated enveloped HEV can be regarded as a nonenveloped HEV.

Recent reports have revealed the nature of the virion-associated ORF2 protein: it has an intact C-terminal end, is nonglycosylated [20], and is translated from the second methionine $16\left(\mathrm{M} 16 ; \mathrm{ORF}^{\mathrm{c}}\right)$ [26] and / or starts with $\mathrm{L} 14\left(\mathrm{ORF}_{\mathrm{i}}\right)$ [20]. However, the precise nature of the fecal nonenveloped HEV virion-associated ORF2 protein is poorly understood.

In the present study, we show that the ORF2 protein constituting both the fecal HEV virion and the detergent (sodium deoxycholate [DOC])- and protease-treated enveloped $\mathrm{HEV}$ are C-terminally truncated. We predicted the protease digestion sites in the truncated ORF2 (ORF2tr) protein. In addition, our structure prediction suggests the digested region of the ORF2tr protein in the fecal HEV virion. Our results reveal that the ORF2 protein in the fecal HEV virion differs from that of the enveloped HEV virion with an intact ORF2 C-terminus.

\section{Results}

\subsection{The C-Terminal Region of the HEV Capsid (ORF2) Protein Is Truncated in Human Feces}

When subjected to sodium dodecyl sulfate-polyacrylamide gel electrophoresis (SDSPAGE) and immunoblotting, the secreted glycosylated ORF2 proteins and the virionassociated ORF2 proteins in the culture supernatant of the genotype 3b HEV (JE03-1760F)producing PLC/PRF/ 5 cells were detected in approximately 80- and 68-kDa forms, respectively (Figure 1), consistent with previous reports [20,26]. However, in contrast to the ORF2 proteins in the culture supernatant (glycosylated and dimerized) and HEVcc, the ORF2 protein of the HEV virion in feces was detected in a 60-kDa form (Figure 1). Similar to the ORF2 protein in the fecal HEV, the detergent $(0.5 \% \mathrm{DOC})$-treated protease $(0.5 \%$ trypsin)-digested HEV cc virion-associated ORF2 protein was also detected in a 60-kDa form (Figure 1). Both of these ORF2 proteins are described as "ORF2tr" in this manuscript.

Xing et al. [17] reported that the C-terminal region of the ORF2 protein is located at the surface of the native virion-sized $T=3 \mathrm{VLP}$ of HEV, consisting of an HEV RNA fragment and the 180 ORF2 proteins without the $\mathrm{N}$-terminal 13 residues. Other studies also report that the C-terminal regions of the ORF2 proteins were located at the surface of VLP $[16,18,27]$. Furthermore, our previous study indicated that the antihuman HEV ORF2, Mab (H6225), was able to capture the HEV virions in feces and the DOC-treated trypsin-digested HEVcc virion and inhibited the infection of cells with fecal HEV and de-enveloped HEVcc in vitro [28]. 

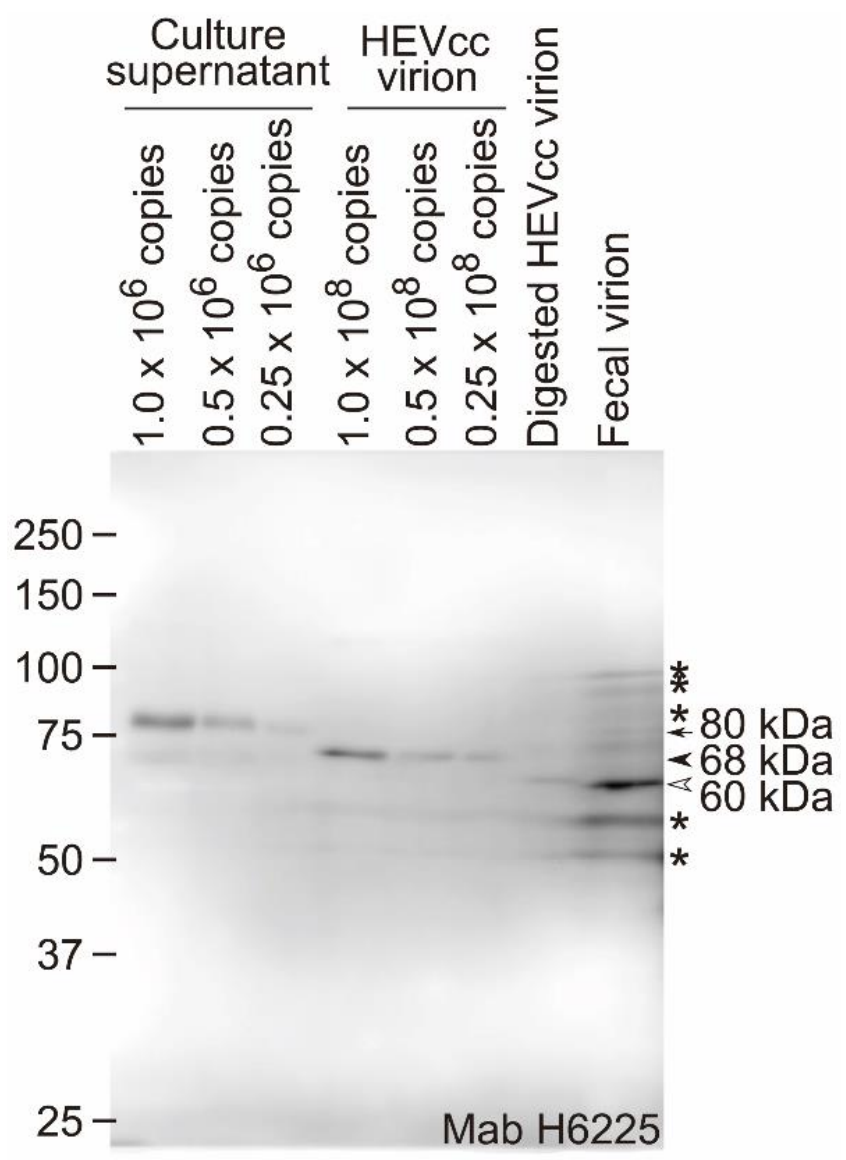

Figure 1. Molecular size of the ORF2 protein in feces. The difference in the molecular sizes of the ORF2 protein of the human HEV virion in the culture supernatant and the human fecal sample. The culture supernatant $(2.5,1.3$, and $0.63 \mu \mathrm{L}$ of culture supernatant, containing $1.0,0.5$, and $0.25 \times 10^{6}$ copies of HEV, respectively, per lane), the ultracentrifuged HEVcc virions in the culture supernatant $\left(1.0,0.5\right.$, and $0.25 \times 10^{8}$ copies of HEV, derived from 250,125 , and $63 \mu \mathrm{L}$, respectively, of culture supernatant/lane) of the HEV-producing cells, the $0.5 \%$ DOC- and $0.5 \%$ trypsin-treated HEVcc virions in the culture supernatant $\left(0.5 \times 10^{8}\right.$ copies of HEV, derived from $125 \mu \mathrm{L}$ of culture supernatant: digested virion), and the ultracentrifuged nonenveloped HEV virion $\left(1.0 \times 10^{8}\right.$ copies of HEV: fecal virion) in the fecal sample from an HEV patient were subjected to SDS-PAGE, and were detected with the anti-HEV mouse monoclonal antibody H6225. Arrow, secreted glycosylated and dimerized ORF2 protein; arrowhead, ORF2 protein associated with enveloped HEV; hollow arrowhead, truncated ORF2 associated with nonenveloped HEV; asterisk, nonspecific signals.

In the present study, we found that the H6225 Mab recognizes the G551-A608 sequence of the ORF2 protein by epitope mapping (Figure 2). Therefore, to confirm that the protease digestion in the HEV virion occurs at the C-terminal region of the ORF2 protein, we constructed a recombinant HEV JE03-1760F/P10 genome (P10_ORF2-FLAG) to produce HEV-like particles with the C-terminally FLAG-tagged ORF2.

However, probably because the inserted FLAG sequence disrupted the essential RNA sequence for HEV genomic RNA replication [29-31], P10_ORF2-FLAG was not grown in the cultured cells. To solve this issue, we inserted the 7091-7151 sequence (60 bases) of the HEV JE03-1760/P10 genome, corresponding to the $3^{\prime}$-terminal end sequence of the ORF2 protein, between the stop codon after the FLAG tag coding sequence, and the authentic stop codon of the ORF2 coding sequence (Figure 3A). After transfection of PLC/PRF/ 5 cells with RNA transcripts of the modified FLAG-tagged JE03-1760F/P10 genome (P10_ORF2-FLAG +60r), FLAG-tagged HEV-like particles were successfully produced and released into the culture media, similar to the parental HEV, JE03-1760F/P10 (P10-wt), and in contrast to the P10_ORF2-FLAG FLAG-tagged ORF2 coding sequence without the 7092-7151 (60 bases) 
repeat (Figure 3B). The obtained FLAG-tagged HEV-like particles were then pelleted down and treated with $0.5 \%$ DOC, before being pulled down with anti-FLAG M2 magnetic beads (binding efficiency: $\approx 97 \%$, Figure $3 \mathrm{~A}$ ); this process resulted in the cell-culture-generated FLAG-tagged HEV-like particle, having the ORF2 protein with an uncleaved C-terminus.
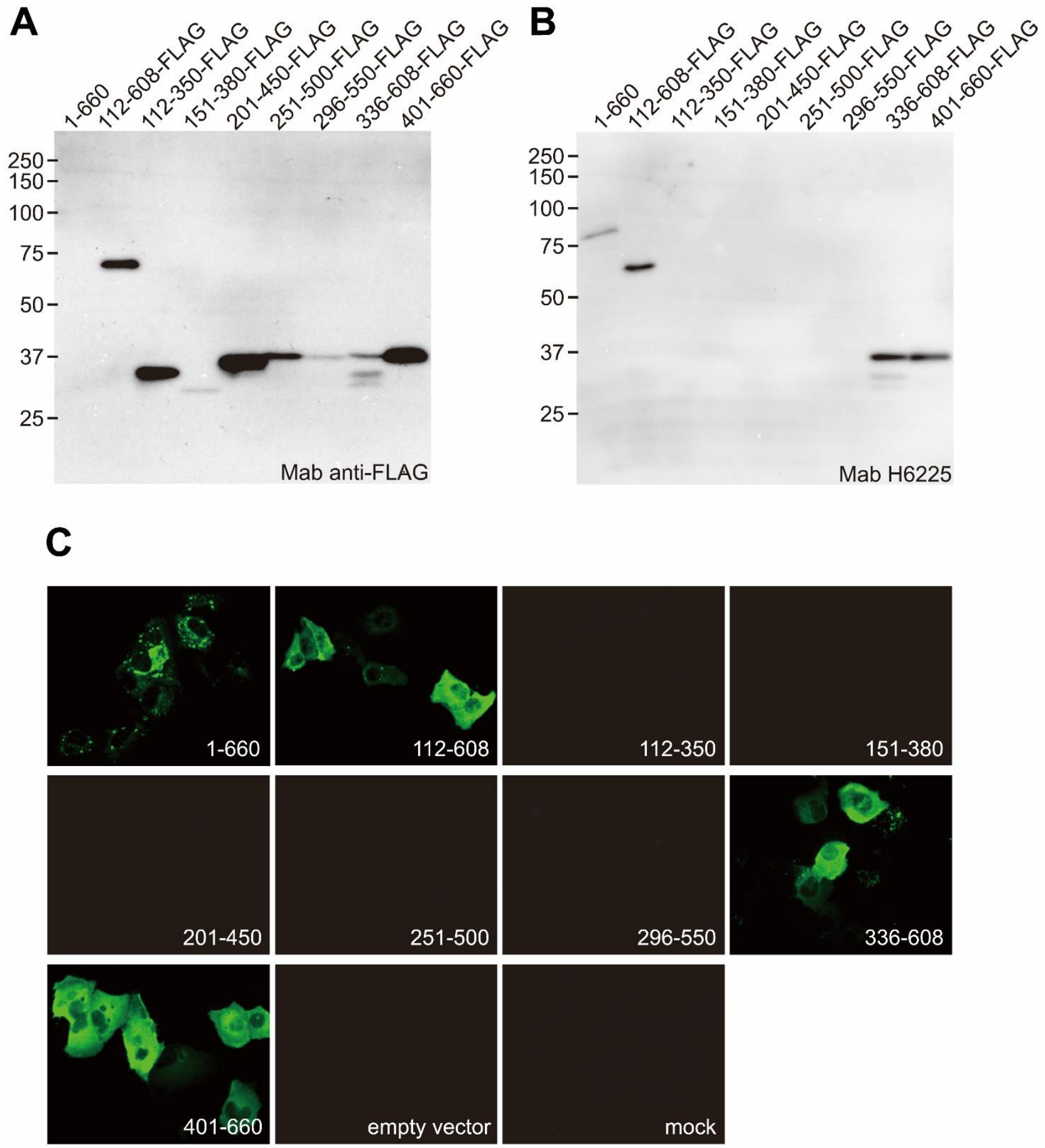

Figure 2. The epitope mapping of H6225, the anti-HEV ORF2 mouse monoclonal antibody. The ORF2 full length and FLAG-tagged deletion constructs were transfected to PLC/PRF/ 5 cells, lysed, and subjected to SDS-PAGE. Blotted membranes were probed with anti-FLAG (A) H6225 anti-HEV mouse monoclonal antibodies (B). (C) An immunofluorescence analysis with H6225 Mab. The cells transfected for the constructs were stained with H6225 Mab. The recognition site of the H6225 Mab was mapped between G551-A608 of the ORF2 protein. 
A

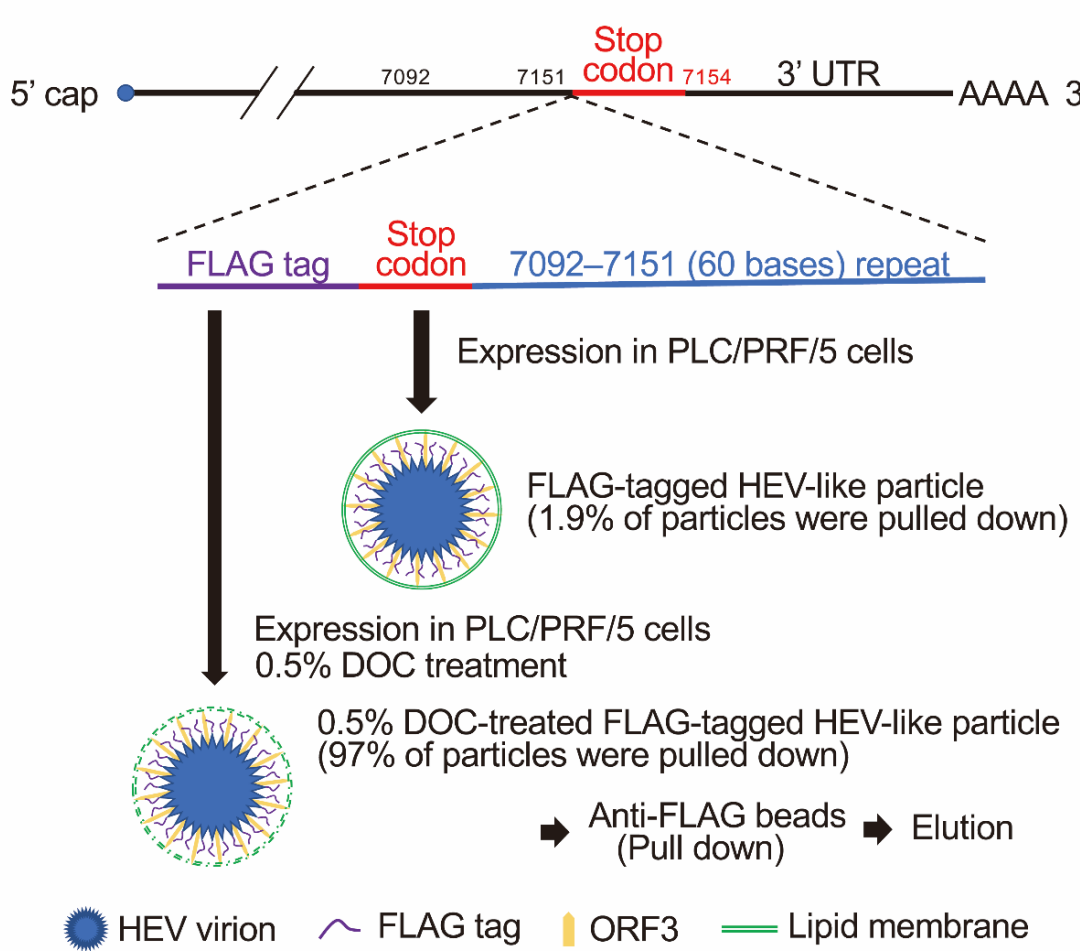

B

C
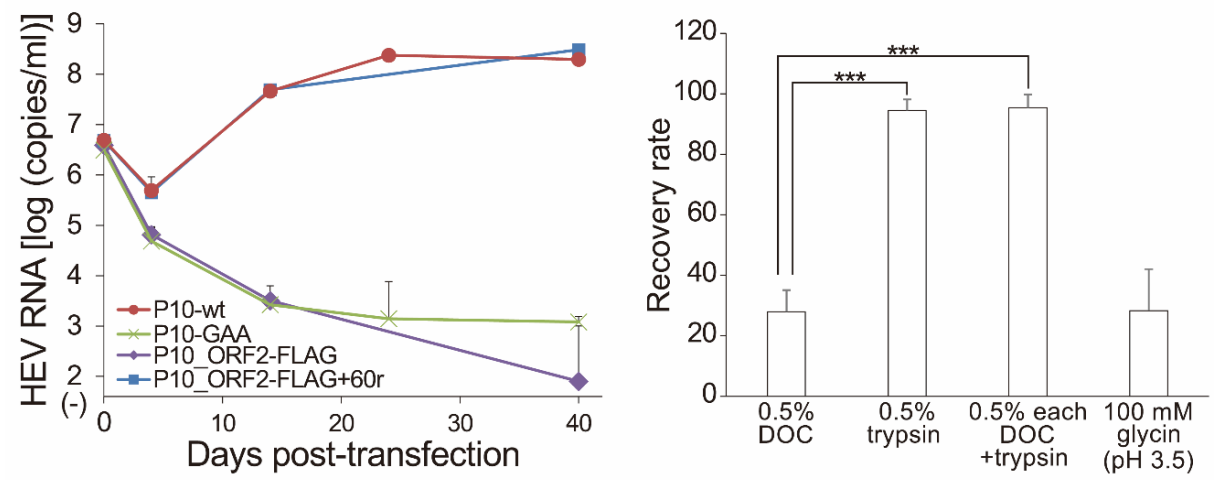

Figure 3. Pull-down of FLAG-tagged HEV-like particle and its elution with trypsin. (A) The schematic image of the FLAG-tagged HEV (genotype 3, JE03-1760F/P10)-like particle construct, expression, and pull-down with anti-FLAG magnetic beads. The FLAG (DYKDDDDK) tag was inserted into the C-terminal end of the ORF2-protein-coding region. In addition, the 7092-7151 (60 bases) repeat of the JE03-1760F/P10 genome (essential region for viral replication) was inserted between the stop codon after the inserted FLAG tag sequence and the authentic stop codon for the ORF2 coding sequence. The expressed HEV-like particle contained a FLAG tag at the C-terminal end of the ORF2 protein. The culture supernatant containing the FLAG-tagged HEV-like particles (P10_ORF2-FLAG-60r) was treated with $0.5 \%$ DOC and captured with anti-FLAG magnetic beads and then eluted, with the HEV RNA genome in the eluent subsequently measured. (B) The growth curve of FLAG-tagged HEV-like particles. The synthesized RNA genomes, the FLAG-tagged ORF2 coding JE03-1760F/P10 RNA, with (P10_ORF2-FLAG + 60r) or without (P10_ORF2-FLAG) the 7092-7151 (60 bases) repeat, and with P10-wt RNA as a positive control and P10-GAA RNA as a negative control for growth, were transfected to PLC/PRF/5 cells. The growth of the wild-type and modified HEV-like particles was monitored with RT-qPCR and plotted $(n=3)$. Error bars represent the mean \pm SD. (C) The captured FLAG-tagged HEV-like particles were eluted with $0.5 \%$ DOC, $0.5 \%$ trypsin, both $0.5 \%$ DOC and $0.5 \%$ trypsin, and $100 \mathrm{mM}$ glycine ( $\mathrm{pH} 3.0$ ). Error bars represent the mean $\pm \mathrm{SD} .{ }^{* * *} p<0.001$ by an ANOVA with the Tukey-Kramer test $(n=3)$. 
The bound FLAG-tagged HEV-like particles were significantly more efficiently eluted by $0.5 \%$ trypsin digestion, with or without $0.5 \%$ DOC, compared to $0.5 \%$ DOC elution only (Figure 3C). This result indicates that the FLAG-tagged HEV-like particles were de-enveloped by DOC, and that the C-terminally FLAG-tagged ORF2 protein lost its C-terminal region with the FLAG tag after digestion by trypsin.

\subsection{Protease Digestion Sites of the ORF2 Protein Associated with the HEV Virion}

To test whether or not other typical proteases ( $\alpha$-chymotrypsin and elastase) secreted in the intestine digested HEV virion, we treated HEVcc virion with $0.5 \%$ DOC, and then $0.5 \%$ elastase, or $0.5 \% \alpha$-chymotrypsin (or with $0.5 \%$ trypsin as a control). After digestion with trypsin, elastase, and $\alpha$-chymotrypsin, the molecular sizes of the ORF2 protein (originally $68.9 \mathrm{kDa}$ ) of the HEVcc virion changed to $60.8,60.8$, and $62.9 \mathrm{kDa}$, respectively, as estimated with a molecular size marker (Figure 4A).

To estimate the sites digested by trypsin, elastase, or $\alpha$-chymotrypsin in the C-terminal region of the ORF2 protein, we aligned the ORF2 amino acid sequences (aa 560-660) of four representative HEV strains (genotype 3b, JE03-1760F, which is used in this study; genotype 1b, SAR-55; genotype 3a, Kernow-C1; genotype 4, HE-JF5/15F). On the basis of the reported protease digestion sites [32-34], R578, A579, and L601 were estimated to be the trypsin, elastase, and $\alpha$-chymotrypsin digestion sites of the ORF2 protein, respectively (Figure 4B). The R578 residue of the ORF2 protein was conserved among $180 \mathrm{HEV}$ strains of genotypes 1-8 (Supplementary Table S1), suggesting that the ORF2 protein of fecal HEV was digested at R578 (furthest away from the C-terminus among the three typical proteases in the intestine), and probably lacked the $88 \mathrm{C}$-terminal amino acid residues (V579-S660 fragment), consistent with the length of the DOC-treated trypsin-digested ORF2 protein (ORF2tr) observed on SDS-PAGE (Figure 4A).

To visualize the possible digestion sites, we next predicted the V470-S660 structure (core of the P domain in Figure 4C) of the ORF2 protein of genotype 3b HEV (JE03-1760F) using the AlphaFold2 software program (Figure 4D). The P domain of the predicted structure almost matched with the previously reported HEV VLP comprising ORF2 structures, 3HAG (genotype 4) [16], 2ZTN (genotype 3) [18], and 6LAT (genotype 1) [27] (Figure 5). The C-terminal disordered region in the previous study [17] was predicted to be four $\alpha$-helices and loop conformations (Figures 4D and 5). In Figure 4D, the highlighted amino acids in red and cyan are the predicted trypsin digestion site, R578, and the predicted $\alpha$-chymotrypsin digestion site, L601, respectively, with amino acid sequences of V579-S660 (yellow) and A574-G576 (green). The V579-L601 has two $\beta$-strands interacting with $\beta$-sheets ( $\beta$-barrel; Figure 4D), which are possibly unstable because of trypsin digestion.

Our results thus suggest that the HEV virion-associated ORF2 proteins are digested with trypsin, elastase, and $\alpha$-chymotrypsin, and that they lack at least A602-S660, the region with four $\alpha$-helices connected by fluctuating loops, and also possibly V579-L601. 
A

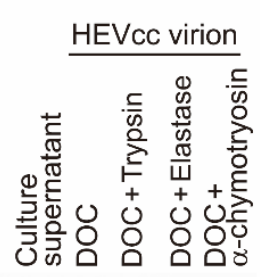

250-

$150-$

$100-$

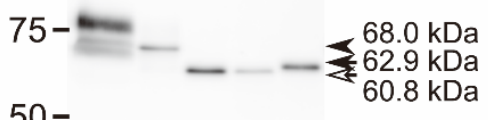

$50-$

$37-$

$25-$

Mab H6225

B

C

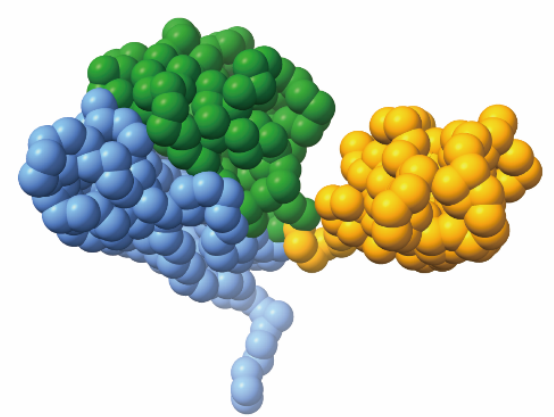

D

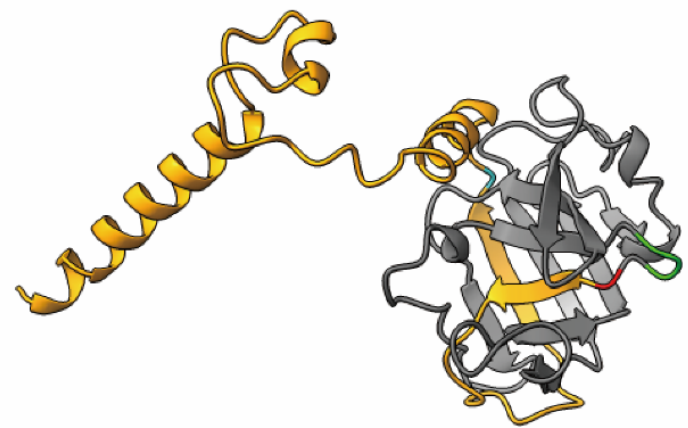

R Trypsin digestion site

$\mathrm{V}$ Elastase digestion site

L $\alpha$-chymotrypsin digestion site
560
570
580
590
600

JE03-1760F (3b) NYNTTASDQ I L I ENAAGHRVA I STYTTSLGAGPTS I SAVGVLAPHSALAV

SAR-55 (1b) NYNTTASDQL L VENAAGHRVA I STYTTSLGAGPVS I SAVAVLAPHSVLAL

Kernow-C1 (3a) NYNTTASDQ I L I ENAAGHRVA I STYTTSLGAGPTS I SAVGVLAPHSALAV

HE-JF5/15F (4) NYNTT VSDQ I L I ENAAGHRVC I STYT TNLGSGPVS I SAVGVLAPHSALAA

$60.6 \mathrm{kDa}$

$62.7 \mathrm{kDa}$

'610 $\quad$ "620 $\quad$ "630 $\quad$ "640 $\quad$ '650

JE03-1760F (3b) LEDTTDYPARAHTFDDFCPECRTLGLQGCAFQST I AELQRL KMKVGKTRES

SAR-55 (1b) LEDTMDYPARAHTFDDFCPECRPLGLQGCAFQSTVAELQRLKMKVGKTREL

Kernow-C1 (3a) LEDTVDYPARAHTFDDFCPECRT LGLQGCAFQST I AELQRLKMKVGKTRES

HE-JF5/15F (4) LEDTVDYPARAHTFDDFCPECRALGLQGCAFQSTVAELQRLKMEVGKTREY

$69.3 \mathrm{kDa}$

Figure 4. Protease digestion site in the ORF2 protein. Trypsin digests human HEVcc (JE03-1760F strain) virion between R578 and V579. (A) The culture supernatant $(2.5 \mu \mathrm{L}$ of culture supernatant containing $1.0 \times 10^{6}$ copies of HEV/lane), the $0.5 \%$ DOC-treated, $0.5 \%$ DOC-treated, and $0.5 \%$ trypsin-digested; $0.5 \%$ DOC-treated and $0.5 \%$ elastase-digested; and $0.5 \%$ DOC-treated and $0.5 \% \alpha$-chymotrypsin-digested HEVcc virions $\left(1.0 \times 10^{8}\right.$ copies each of ultracentrifuge-purified HEV derived from $250 \mu \mathrm{L}$ of culture supernatant/lane) were subjected to SDS-PAGE, and detected with the anti-HEV mouse monoclonal antibody, H6225. Arrowhead, arrow, and hollow arrow indicate full-length ORF2, $\alpha$-chymotrypsin-digested ORF2, and trypsin- or elastase-digested ORF2 proteins, respectively. Molecular weights were estimated with the size marker. (B) The alignment of 560-660 amino acids of the ORF2 protein sequences from the genotypes, 3b (JE03-1760F), 1b (SAR-55), $3 \mathrm{a}$ (Kernow-C1), and 4 (HE-JF5/15F). Arrows indicate the calculated molecular weights: M16-R578, 60.6 kDa; M16-L601, 62.7 kDa; M16-S660, 69.3 kDa. (C) The structure of the amino acids 118-06 of the ORF2 protein (3IYO). The S (shell, 118-313), M (middle, 314-453), and P (protruding, 455-end) domains are highlighted in blue, green, and orange, respectively. (D) The structure of the $\mathrm{P}$ domain and C-terminal region (P461-S660) of the genotype 3b HEV (JE03-1760F) ORF2 protein was predicted using the AlphaFold2 software program. The A574-G576, R578 residue, V579-S660, and L601 are highlighted in green, red, yellow, and cyan, respectively. 


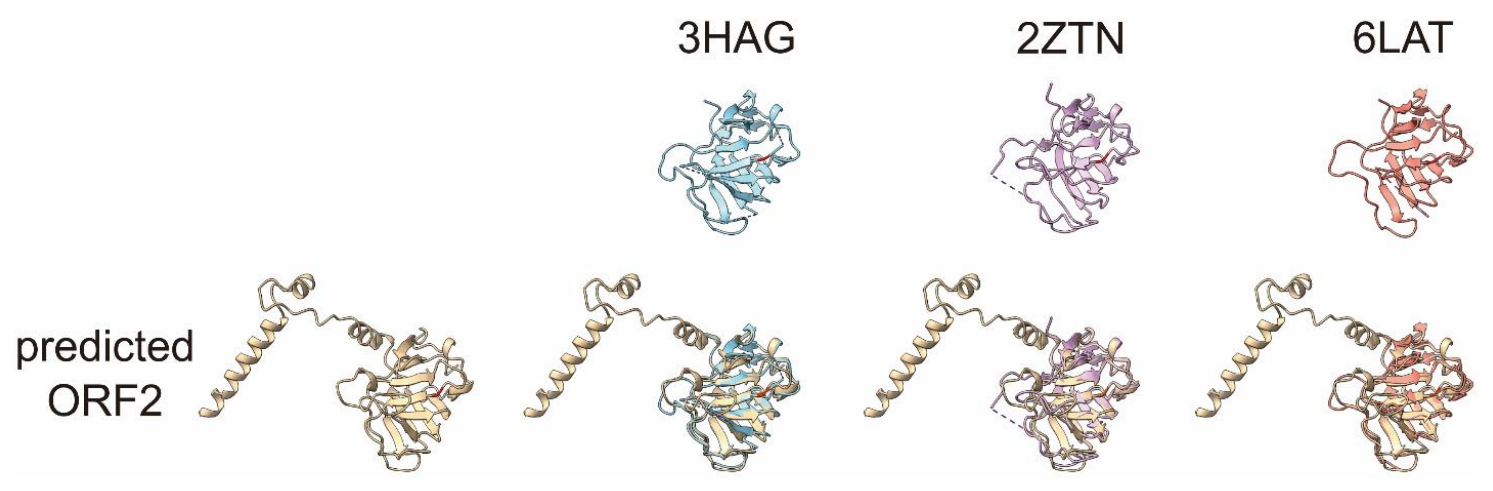

Figure 5. The superimposed image of the AlphaFold2-predicted ORF2 and previously reported ORF2 protein structures. The AlphaFold2-predicted ORF2 protein (amino acids 461-660) structure and the previously reported ORF2 protein structure, 3HAG (amino acids 461-605 [16]), 2ZTN (amino acids 461-606 [18]), and 6LAT (amino acids 461-605 [27]) were superimposed using the UCSF ChimeraX software program, ver. 1.1. The R578 residue is highlighted in red. Dotted line represents disordered region.

\subsection{The ORF2 Protein of Rat HEV in Feces Is C-Terminally Truncated, Similar to That of the Fecal HEV in Humans}

To investigate whether or not the ORF2 proteins of rat HEV in feces are also Cterminally truncated, we prepared a fecal rat HEV suspension and subjected it to Western blotting. The molecular size of the fecal rat HEV-associated ORF2 protein was $67.3 \mathrm{kDa}$, which is similar to that of the $0.5 \%$ DOC-treated and $0.5 \%$ trypsin-digested ORF2 protein of rat HEVcc (Figure 6A). The typical proteases secreted in the intestine, trypsin, elastase, and $\alpha$-chymotrypsin truncated the ORF2 protein of rat HEVcc (Figure $6 \mathrm{~B}$ ), and were estimated to digest it at residues K567, A568, and L589, respectively (Figure 6C). The K567 residue of the ORF2 protein is conserved among various rat HEV strains (Supplementary Table S2).

To visualize the trypsin digestion site, we predicted the structure of the rat HEV (ratELOMB-131L) ORF2 protein using the AlphaFold2 software program. The two $\beta$ strands followed by the trypsin digestion site, K567, highlighted in red and interacting with other $\beta$-sheets, were considered unstable because of the trypsin digestion (Figure 6D). Thus, the three $\alpha$-helices at the $C$-terminal end, and possibly the two $\beta$-strands in the $P$ domain of the rat HEV ORF2 protein, were considered to be lost in the feces, similar to the findings of the fecal HEV virion in humans.

These data collectively indicate that the ORF2 proteins associated with rat HEV in feces, and those of rat HEVcc treated with detergent and protease, are C-terminally truncated (ORF2tr).

\subsection{The Predicted Structures of Nondigested and Digested HEV Virions}

Previously, Xing et al. [17] reported the X-ray model of a $T=3$ icosahedral VLP, containing the HEV RNA genome fragment, with a cryo-electron microscope (cryo-EM, PDB ID: 3IYO; aa 118-606). Because the AlphaFold2-predicted ORF2 protein P-domain structure is almost the same as the previously reported ORF2 protein P domains (Figure 5), we replaced the P domain, V470-A606, of the 3IYO, with the V470-A606 of the AlphaFold2predicted ORF2 protein structure, as 3IYO consists of only $\mathrm{C} \alpha$ atoms (Figure 7A). We then superimposed this VLP and the AlphaFold2-predicted ORF2 structure (Figure 4D) as a predicted de-enveloped and nondigested HEV virion. The superimposed AlphaFold2predicted ORF2 protein structures (V470-A606) almost matched with those of 3IYO (data not shown). The A602-A606 in Figure 7A was replaced with the A602-S660 of the AlphaFold2predicted ORF2 (Figure 7B). In addition, we displayed the de-enveloped trypsin-digested HEV virion structure, which lost V579-S660 (highlighted in blue in Figure 7B). The H6225 $\mathrm{Mab}$ recognizes the region (highlighted in green) on the surface of the predicted trypsindigested virion (Figure 7C). This predicted model supports the finding that H6225 Mab can capture fecal HEV [28]. Therefore, it is likely that the predicted HEV virion (Figure 7B) 
is digestible by proteases into smaller particles (Figure 7C), which H6225 Mab is able to capture, indicating that the smaller particles (Figure 7C) contain the H6225 Mab recognition site of the ORF2 protein (G551-A608).

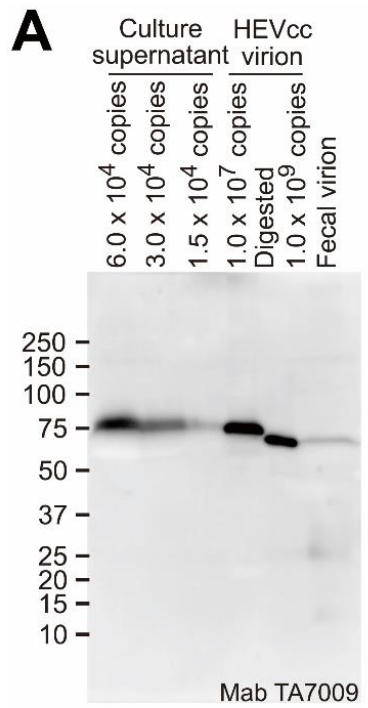

C
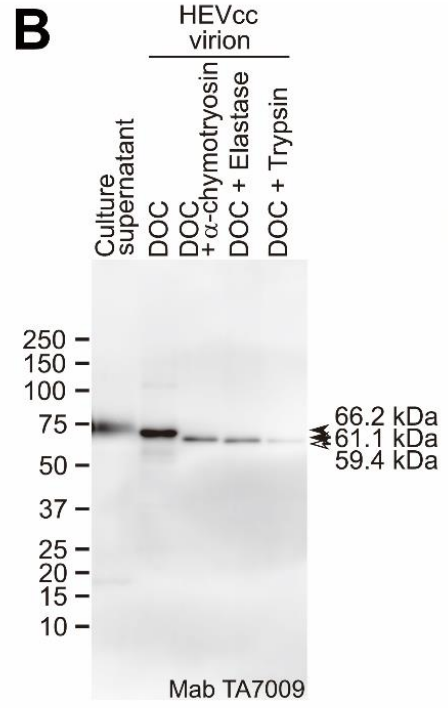

D

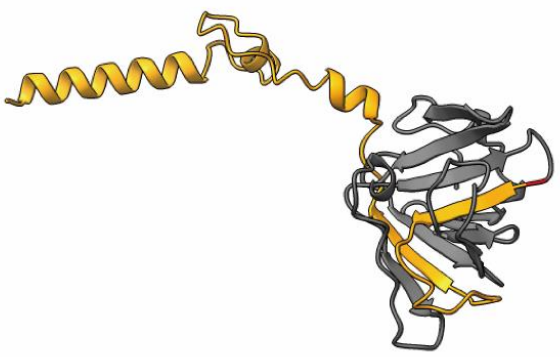

K Trypsin digestion site

A Elastase digestion site

L $\quad \alpha$-chymotrypsin digestion site

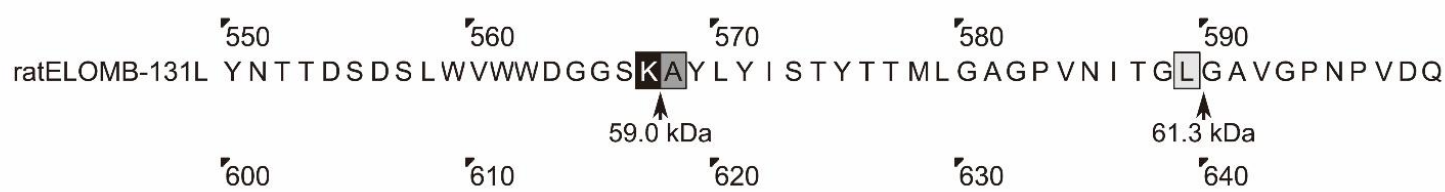

ratELOMB-131L AS A V VPMHNL QDGCPACALAGLSSCL VADAMAR VQAL SAAAADS V

$66.6 \mathrm{kDa}$

Figure 6. Molecular size of the ORF2 protein of rat HEV in feces. The truncated rat ORF2 protein in the rat fecal sample and the protease-digested rat HEV virion. (A) The culture supernatant $\left(4.8,2.4\right.$, and $1.2 \mu \mathrm{L}$ of culture supernatant, containing $6.0,3.0$, and $1.5 \times 10^{4}$ copies of HEV, respectively, per lane), the ultracentrifuged $\mathrm{HEVcc}$ virion $\left(1.0 \times 10^{7}\right.$ copies of $\mathrm{HEV}$, derived from $800 \mu \mathrm{L}$ of culture supernatant/lane), the $0.5 \%$ DOC- and $0.5 \%$ trypsin-treated ultracentrifuged HEVcc virion $\left(1.0 \times 10^{9}\right.$ copies/lane: digested), and the ultracentrifuged nonenveloped HEV virion $\left(1 \times 10^{9}\right.$ copies/lane: fecal virion) in the fecal sample from rat HEV (ratELOMB-131L)-inoculated immunodeficient rats were subjected to SDS-PAGE, and were detected with the antirat HEV mouse monoclonal antibody TA7009. The estimated molecular weights of the ORF2 proteins were approximately $67 \mathrm{kDa}$ for both the $0.5 \%$ DOC- and $0.5 \%$ trypsin-treated HEVcc and the fecal HEV. (B) The culture supernatant $\left(1.2 \times 10^{4}\right.$ copies of $\mathrm{HEV}$, derived from $1.2 \mu \mathrm{L}$ of culture supernatant/lane), the ultracentrifuged $\mathrm{HEVcc}$ virion $\left(1.0 \times 10^{7}\right.$ copies of $\mathrm{HEV}$, derived from $1.0 \mathrm{~mL}$ of culture supernatant/lane), the $0.5 \%$ DOC-treated and $0.5 \% \alpha$-chymotrypsin-digested, $0.5 \%$ DOC-treated and $0.5 \%$ elastase-digested, and $0.5 \%$ DOC-treated and $0.5 \%$ trypsin-digested HEVcc virions were subjected to SDS-PAGE, and were detected with the anti-HEV mouse monoclonal antibody, TA7009. Arrowhead, arrow, and hollow arrow indicate full-length ORF2 (66.2 kDa), $\alpha$-chymotrypsin-digested ORF2 (61.1 kDa), and trypsin- or elastase-digested ORF2 proteins (59.4 kDa), respectively. Molecular weights were estimated with the size marker. (C) The amino acid sequence of the Y550-V644 amino acids of the rat HEV ORF2 protein. Arrows indicate the calculated molecular weights: K567, 60.6 kDa; L589, 62.7 kDa; and V644, 69.3 kDa. (D) The structure of the P domain and C-terminal region (I451-V644) of the rat ORF2 protein structure was predicted with the AlphaFold2 software program. The K567 residue and A568-V644 residues are highlighted in red and yellow, respectively. 
A

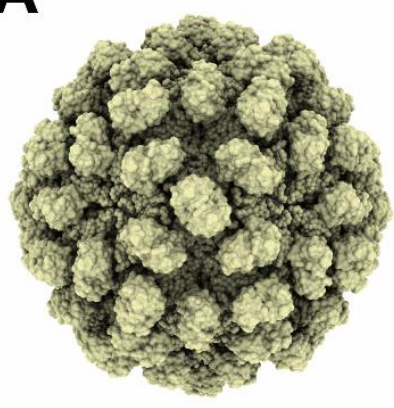

C

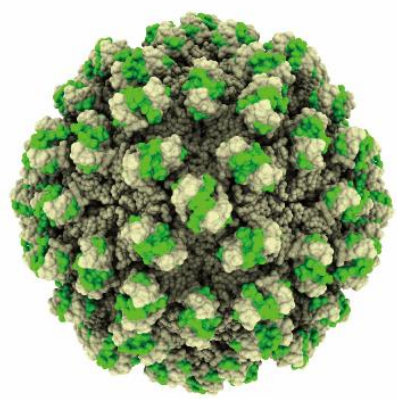

B

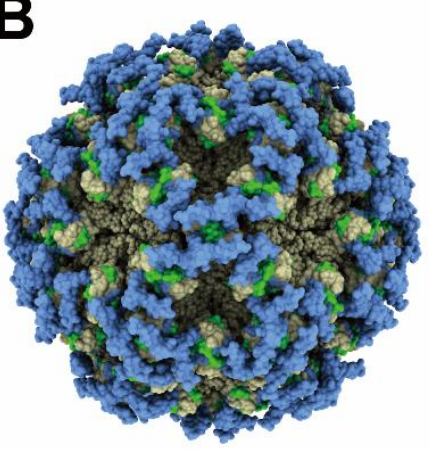

D

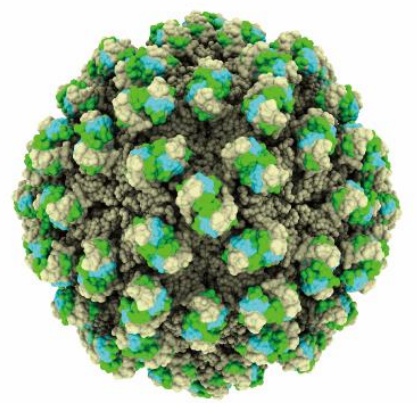

Figure 7. Protease-digested HEV virion structure. A predicted nonenveloped and nondigested HEV and nonenveloped and trypsin-digested virion structure. (A) Model-fitting image of the previously reported X-ray model of T = 3 VLP (PDB ID: 3IYO). The V470-A606 is replaced by the V470-A606 of the AlphaFold2-predicted ORF2 structure. (B) Superimposed image of 3IYO and the AlphaFold2predicted ORF2 structure. The V470-A606 is replaced by the V470-S660 of the AlphaFold2-predicted ORF2 structure. The H6225-antibody-recognizing region, G551-R578, and the C-terminal region cleaved by trypsin V579-S660 are highlighted in green and blue, respectively. (C) The H6225-antibodyrecognizing region, G551-R578, is highlighted in green. (D) The G551-R578 region and the V579-L601 region, which may be retained on the surface of fecal HEV or HEVcc, after treatment with detergent and trypsin, because of the interaction between the two $\beta$-strands (579-582; and 595-600) and other $\beta$-sheets (492-495 and 568-572; and 470-476 and 546-549, respectively), are highlighted in green and cyan, respectively.

\section{Discussion}

The present study reveals that the molecular size of both the human and rat ORF2 proteins associated with the fecal HEV virions (ORF2tr) were smaller than the ORF2 proteins (aa 14/16-660 [20,26] in human HEV, and probably aa 21 [2 methionine]-644 in rat $\mathrm{HEV}$ ) associated with the enveloped HEVcc virions. Sodium deoxycholate and typical proteases, including trypsin, elastase, and $\alpha$-chymotrypsin, all secreted in the intestine, were found to de-envelope and digest enveloped HEV virions into smaller nonenveloped particles consisting of ORF2tr proteins. The structural prediction study on the human HEV ORF2 protein suggests that at least the A602-S660 fragment, which has four $\alpha$-helixes and does not participate in the structure of the $\mathrm{P}$ domain, is dissociated from the HEV virions in feces.

In this study, to generate an HEV-like particle with a FLAG-tagged ORF2 protein, we inserted the nucleotide sequence corresponding to the FLAG tag (DYKDDDDK) and the 7092-7151 (60 bases) repeat into the parental genotype 3b HEV JE03-1760F/P10 genome (Figure 3A). One stem-loop structure of the cis-reactive element (CRE) overlaps with the $3^{\prime}$-end of the ORF2-coding region and the $3^{\prime}$-terminal sequence [29]. Because the insertion of the FLAG-tag sequence disrupted the secondary structure harboring the CRE, the HEV-like particle, with the genome having the FLAG-tagged ORF2 sequence but not the 7092-7151 repeat, did not grow in cell culture (Figure 3B). These results corroborate 
previous findings by confirming that the secondary structure harboring the CRE in the $3^{\prime}$-terminus is important for replicating the HEV genome [29-31]. In addition, we inserted the EGFP sequence into the JE03-1760F/P10 HEV RNA genome instead of the FLAG tag sequence, together with the 7092-7151 repeat, and transfected the recombinant JE031760F/P10 genome into cultured cells, and then monitored the growth of the EGFP-tagged HEV-like particle. Contrary to our expectation, the EGFP-tagged HEV-like particle was not detectable, and even the complete revertant without the EGFP genome sequence was generated in the cell culture (Supplementary Figure S1). These data indicate that the addition of an extra sequence is limited at the $3^{\prime}$-terminal end of the ORF2 coding sequence.

Trypsin, elastase, and $\alpha$-chymotrypsin secreted in the intestine digest the ORF2 protein associated with the HEVcc virion at different sites because of the differences in their recognition sites (Figure 4A,B), especially the trypsin digestion site is R578, which is inside a core of the P domain. In a previous study [16], A574-G576 was reported to be a fluctuating region, according to a cryo-EM analysis (Figure 5), which suggests that trypsin is able to recognize and digest the downstream R578. Among the three typical proteases secreted in the intestine, trypsin digests ORF2 proteins at the C-terminal region into the smallest size, and the resulting protein (ORF2tr) was estimated to have the same size as the ORF2tr protein of the fecal HEV virion (Figure 1). However, in contrast to the ORF2tr, the glycosylated and dimerized ORF2 protein in the culture supernatant was reported to be digested into a smaller size than the ORF2tr protein [35]. Therefore, it is very likely that the DOC-treated trypsin-digested HEVcc virion possesses the same characteristics as fecal HEV.

Xing et al. [17] reported the X-ray model of $T=3$ icosahedral VLP containing an HEV RNA fragment with a cryo-EM (PDB ID: 3IYO). This model contained an ordered structure with D118-A606. We superimposed 3IYO and the AlphaFold2-predicted ORF2 structure to visualize the nonenveloped and nondigested HEV virion (Figure 7B). Because AlphaFold2 predicted that L607-S660 would fluctuate in 3IYO, the four $\alpha$-helices at the C-terminal region were expected not to constitute the ordered domain structure. In addition, we previously reported that H6225 Mab was able to capture the DOC-treated nondigested HEV virion in circulating blood [25] and culture supernatant [23]. Furthermore, the $\alpha$-helices at the C-terminal end were predicted to move vertically with the WebNMA server [36] (available at: http://apps.cbu.uib.no/webnma3/, accessed on 6 December 2021, Figure 8), while other models predicted with WebNMA indicated that the $\alpha$-helices move horizontally (data not shown); thus, these $\alpha$-helices were considered to move freely on the surface of the HEV virion. Since the $\alpha$-helices are fluctuating, a structural analysis (such as X-ray crystallography and NMR) is unable to determine the unique structure of the HEV virion consisting of full-length ORF2 protein. Only structure prediction is able to predict the HEV structure consisting of the full-length ORF2 proteins. Therefore, the combination structure involving the HEV VLP, reported by Xing et al. [17], and our structure, predicted with AlphaFold2, displayed in Figure 7B-D, has important implications in structural studies and interaction analyses.

In the present study, the ORF2 protein constituting the fecal HEV virion was estimated to be cleaved at R578/V579 and L601/A602 by trypsin and $\alpha$-chymotrypsin, respectively. The extreme C-terminal end fragment of A602-S660 seems to have been removed from the surface of the fecal HEV virion, with the following reasons cited: First, this region was not ordered by cryo-EM [17], suggesting that this region does not interact with the surface of the fecal HEV virion after cleavage; second, another anti-ORF2 Mab (H6210) [28], which is mapped at the V609-S660 C-terminal region of the ORF2 protein (Supplementary Figure S2), was not able to capture the fecal HEV virion [28]. In contrast, whether or not the V579-L601 fragment is retained on the virion surface in feces remains unclear. In either case, the G551-R578 region is exposed at the surface of the fecal HEV virion (Figure 7C,D), consistent with the evidence that the H6225 Mab can almost wholly capture the HEV in feces [25,28], and that this Mab recognizes not only the G551-A608 region of the ORF2 
protein (Figure 2), but also the C-terminally trypsin-digested ORF2 protein associated with HEV virion, being devoid of V579-S660 (Figure 4A,B).

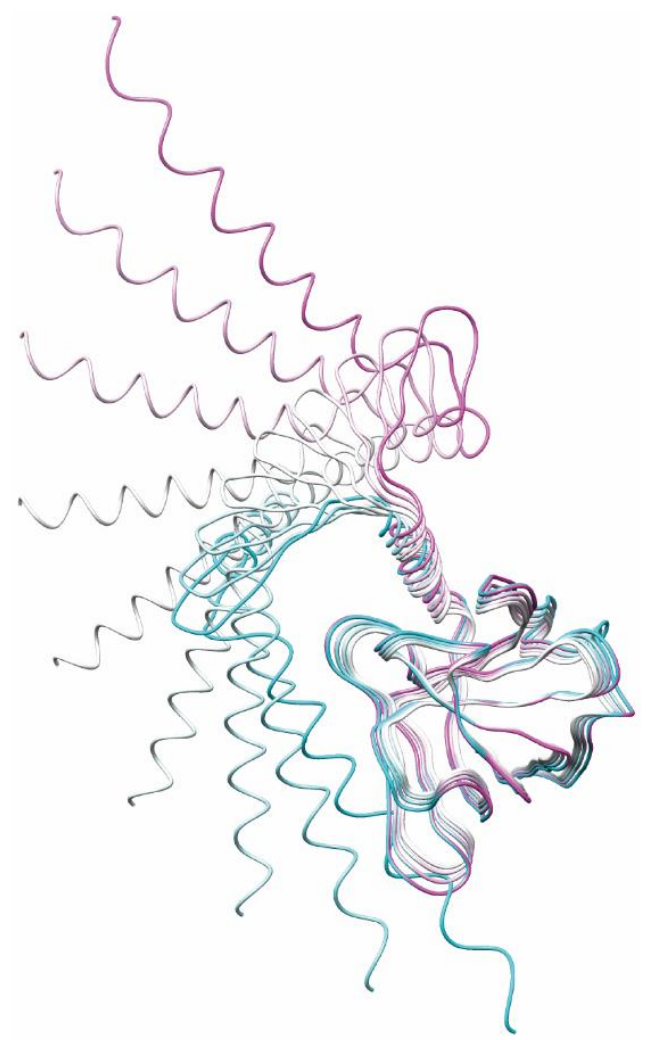

Figure 8. Visualization of the ORF2 protein backbone dynamics. The movement of the V470-S660 of the AlphaFold2-predicted ORF2 protein structure is colored magenta to cyan, determined via a normal mode analysis using the WebNMA server. The V470-L600 is superimposed using the UCSF Chimera software program, ver. 1.15. The $\alpha$-helix at the C-terminal end is predicted to move perpendicular to the surface of the virion.

However, there is a possibility that the V579-L601 region may be retained on the surface of fecal HEV virion (Figure 7D) because of the interaction between the two $\beta$ strands and other $\beta$-sheets ( $\beta$-barrel, Figure $4 \mathrm{C}$ ). Previous studies support this possibility. Arias et al. [37] found in their review that, similar to HEV, human astrovirus (HAstVs) virion is digested by extracellular trypsin during maturation. The 70-kDa immature capsid protein (VP70) that constitutes the immature virion is digested into VP34, VP27, and VP25. The cleaved VP27 and VP25 bind to the mature HAstVs, consisting of VP34, and function as spike proteins. Schofield et al. [38] reported that the Mabs, whose epitopes are mapped at the R578-L607 region of genotype $1 \mathrm{HEV}, \mathrm{SAR}-55$, neutralize the infection of fecal SAR$55 \mathrm{HEV}$ to rhesus monkeys, suggesting the presence of the cleaved R578-L607 fragment. In both cases, the core of the P domain, which is expected to be a spike because of its attachment activity to HEV-susceptible cells [18], is exposed at the surface of the fecal HEV virion, since the A602-S660 region is removed from the surface of the fecal HEV virion.

During the HEV lifecycle, the C-terminus of the HEV ORF2 protein and its digestion play an important role in the virion assembly and infection of nonenveloped HEV. Nonenveloped HEV harboring an intact C-terminus, M16-S660 (ORF2 ${ }^{\mathrm{C}}$ ) [26], and/or L14-S660 $\left(\mathrm{ORF}_{\mathrm{i}}\right)$ [20], is produced in infected cells. Shiota et al. [39] reported that the 52 C-terminal amino acids (V609-S660) of the ORF2 protein of genotype $3 \mathrm{HEV}$ are essential for HEV encapsulation and viral particle stabilization. In our study, the C-terminal region (at least A602-S660) of the ORF2 protein was truncated in nonenveloped HEV by the digestion of proteases. Kalia et al. [40] reported that the C-terminal-truncated VLP (aa 112-607) binds to heparan sulfate proteoglycans (HSPGs) on the cell surface. Whether or not the nonen- 
veloped HEV virion composed of ORF2 proteins with an intact C-terminus binds to HSPGs is unclear at present. In summary, once an HEV virion with ORF2 proteins with an intact C-terminus, and that is coated with the host envelope is produced, it becomes stable. The enveloped HEV is then secreted into the intestine via the biliary tract, and de-enveloped and protease-digested into nonenveloped HEV with C-terminally truncated capsid proteins (ORF2tr). The nonenveloped HEV binds to HSPGs on the surface of the HEV-susceptible cells. However, the putative receptor for nonenveloped HEV is still unknown. On the basis of our finding, that detergent-treated and protease-digested HEVcc shares the same morphological characteristics as fecal HEV, we suggest that future studies be conducted using detergent-treated and protease-digested HEV, mimicking both human and rat fecal HEV, in order to reveal the HEV lifecycle, including the details of virus attachment and internalization.

In conclusion, the present study clarified that both the human and rat ORF2 proteins associated with enveloped HEV have an intact C-terminus, but these ORF2 proteins associated with nonenveloped HEV are C-terminally truncated (ORF2tr). The typical proteases secreted in the intestine digest the virion-associated ORF2 protein into a smaller one, similar to the ORF2tr protein associated with fecal HEV. A structure prediction analysis of the ORF2 protein supported the notion that the C-terminal region of both the human and rat HEV ORF2 proteins are removed by protease digestion. A structural study of the HEV virion shape indicated that the four $\alpha$-helices (A602-S660) move freely on the surface of the HEV virion; however, at least these four $\alpha$-helices are removed from the surface of nonenveloped protease-digested HEV and fecal HEV. On the basis of the findings from the present study concerning the nature of the HEV virion-associated ORF2tr proteins, we conclude that studies on nonenveloped HEV virions can be performed using DOC- and protease-treated $\mathrm{HEVcc}$ virions when fecal samples containing HEV virions are not available. Our study is the first of its kind regarding the maturation of a nonenveloped HEV and has important ramifications in the study of the nonenveloped $\mathrm{HEV}$ infection mechanism.

\section{Materials and Methods}

\subsection{Fecal Suspensions Containing Human and Rat HEV Strains}

For the human fecal sample, we used a 15\% (wt/vol) suspension of feces, obtained at the acute phase, from a Japanese patient who contracted a domestic infection of genotype 3b HEV (JE03-1760F), which was successfully used for the development of a cell culture system for HEV [41]. For the rat fecal sample, we used a 15\% suspension of feces obtained from a nude rat (F344/NJcl-rnu/rnu (CLEA Japan, Inc., Tokyo, Japan)) experimentally infected with a rat HEV strain (ratELOMB-131), which was successfully used to develop a cell culture system for rat HEV [42]. A 15\% fecal suspension in $10 \mathrm{mM}$ phosphate-buffered saline, with a $\mathrm{pH}$ of 7.5 (PBS), was centrifuged at $6200 \times \mathrm{g}$ for $5 \mathrm{~min}$ at $4{ }^{\circ} \mathrm{C}$, and the resulting clear supernatants were aliquoted and stored at $-80^{\circ} \mathrm{C}$ until use. They were then thawed on ice, filtrated through microfilters with a pore size of $0.2 \mathrm{~mm}$ (Millex-GV; Merck Millipore, Billerica, MA, USA), and subjected to assays.

\subsection{Antibodies}

The anti-ORF2 monoclonal antibodies (Mabs), H6225 and H6210 (for human HEV) and TA7009 (for rat HEV), were previously raised against the recombinant ORF2 proteins (aa 111-660 of human HEV ORF2, and aa 101-644 of rat HEV ORF2, respectively) [28,43]. The anti-FLAG M2 magnetic beads were purchased from Merck Millipore.

\subsection{Cell Culture and Inoculation}

PLC/PRF/5 cells (ATCC No. CRL-8024; American Type Culture Collection, Manassas, VA, USA) were cultured as previously described [41]. For inoculation, naïve cells were seeded at $1.0 \times 10^{5}$ cells/well onto a 24-well plate (BioLite 24 Well Multidish; 930186, Thermo Fisher Scientific, Waltham, MA, USA). The next day, the cells were washed and inoculated with a cell-culture-adapted genotype 3b HEV strain (JE03-1760F, passage 26; 
$1.0 \times 10^{5}$ copies/well) as inoculum. The inoculated cells were rinsed with phosphatebuffered saline (PBS) five times to thoroughly wash out the remaining HEV and were then grown in growth medium. As described previously, half of the culture medium was exchanged for fresh growth medium every other day [44].

\subsection{Preparation of Detergent-and/or Protease-Treated HEV Virions}

The human and rat HEV in the culture supernatant and the fecal suspensions $[42,45]$ were centrifuged at 150,000 $\times g$ (Optima TLX; Beckman Coulter Inc, Miami, FL, USA). The resulting pellets of $\mathrm{HEV}$ virions were treated with $0.5 \%$ DOC, and digested with $0.5 \%$ trypsin, $0.5 \%$ elastase (Fuji Film Wako Pure Chemical Co., Tokyo, Japan), or $0.5 \% \alpha$-chymotrypsin (Fuji Film Wako Pure Chemical Co.), for $1 \mathrm{~h}$ at $37^{\circ} \mathrm{C}$. The digested HEV virions were then treated with the serine protease inhibitor, p-APMSF (Merck Millipore).

\subsection{Immunoblotting}

The cells were lysed with $2 \%$ Nonidet P-40 (NP-40) in PBS. The collected culture supernatants, cell lysates, and virions in ultracentrifuged pellets were heated to $70{ }^{\circ} \mathrm{C}$ for $10 \mathrm{~min}$ in SDS sample buffer (final concentration, $60 \mathrm{mM}$ Tris- $\mathrm{HCl}, 2 \% \mathrm{SDS}, 5 \%$ glycerol, $0.2 \%$ bromophenol blue [BPB], 5\% 2-mercaptoethanol [2-ME], and $100 \mathrm{mM}$ dithiothreitol [DTT]). They were separated by SDS-PAGE on an 11/17.5\% step-gradient Anderson gel [46], and then electroblotted onto Immobilon-P membranes (Millipore Corporation, Billerica, MA, USA). The human and rat HEV ORF2 proteins were detected with the H6225 or H6210 mouse antihuman HEV ORF2 protein Mabs [28], or the TA7009 mouse antirat ORF2 protein Mab [43], respectively. The bound primary antibody was detected with horseradish peroxidase (HRP)-conjugated secondary antibody (\#8125; Cell Signaling Technology, Danvers, MA, USA) and SuperSignal West Femto (Thermo Fisher Scientific) HRP substrate.

\subsection{Construction of $\mathrm{N}$-and/or C-Terminally Truncated ORF2 Protein and FLAG-Tagged HEV-Like Particles}

To construct ORF2 protein-expressing recombinant plasmids, we inserted the nucleotide sequence of the JE03-1760F/P10 genome [47] corresponding to the ORF2 protein, which was amplified using specific primers (Supplementary Table S3), into the pCI mammalian expression plasmid vector (Promega, Madison, WI, USA) using the NheI-XbaI sites. To construct the N- and C-terminally truncated ORF2 112-350-FLAG construct, the NheI-112-350-XbaI-FLAG-NotI was amplified and inserted to the NheI-NotI sites of pCI plasmid (pCI-112-350-FLAG). To construct other N-and/or C-terminally truncated ORF2 protein expression plasmid vectors, NheI-112-608-XbaI, NheI-112-350-XbaI, NheI-151-380XbaI, NheI-201-450-XbaI, NheI-251-500-XbaI, NheI-296-550-XbaI, NheI-336-608-XbaI, and NheI-401-660-XbaI of ORF2 were amplified with specific primers (Supplementary Table S3), and then switched with the NheI-112-350-XbaI of pCI-112-350-FLAG using the NheI-XbaI sites, and were inserted into a pCI plasmid vector using the NheI-XbaI site. The plasmid vectors were transfected to PLC/PRF/ 5 cells using TransIT ${ }^{\circledR}$-LT1 Reagent (TAKARA, Kyoto, Japan).

The nucleotide sequence corresponding to the FLAG tag was inserted into the Cterminal end of the ORF2 coding sequence of an infectious full-length HEV cDNA clone (pJE03-1760F/P10) [47]. Furthermore, the 7091-7151 sequence of the JE03-1760/P10 genome (60 bases, $3^{\prime}$ end of the ORF2 coding region) was inserted after the FLAG peptide coding sequence to maintain the RNA secondary structure in the $3^{\prime}$-terminal sequence, containing two stem-loop structures of cis-reactive elements (CREs) in the HEV genome (P10_ORF2FLAG + 60r) [30]. In addition, the FLAG-tagged ORF2 coding sequence without the 7092-7151 (60 bases) repeat RNA sequence was constructed (P10_ORF2-FLAG). As positive and negative controls for viral growth, the parental JE03-1760F/P10 (P10-wt) and JE031760F/P10-GAA (P10-GAA) with a GAA mutation in the ORF1 protein (as an amino acid sequence: 1560GDD1562 to GAA of JE03-1760F/P10) were used, respectively. We fused the DNA fragments amplified with specific primers (Supplementary Table S3) to construct 
these plasmids using an In-Fusion HD cloning kit (Z9633N; TaKaRa Bio Inc., Shiga, Japan), according to the manufacturer's protocol.

\subsection{In Vitro Transcription, Capping, and Transfection}

P10-wt, P10-GAA, P10_ORF2-FLAG, or P10_ORF2-FLAG+60r plasmids were linearized with BamHI-HF (R0136; New England Biolabs Inc., Ipswich, MA, USA). The linearized plasmids were subjected to the synthesis of P10-wt, P10-GAA, P10_ORF2-FLAG, and P10_ORF2-FLAG+60r RNA with an AmpriScribe ${ }^{\mathrm{TM}}$ T7-FlashTM Transcription Kit (ASF3507; epicentre/Illumina, Inc., San Diego, CA, USA), and were then purified and capped with a ScriptCap ${ }^{\mathrm{TM}}$ m7G capping system (C-SCCE0625; CELLSCRIPT, Madison, WI, USA), according to the manufacturer's protocol. The synthesized RNAs were transfected to PLC/PRF/5 cells with a TransIT-mRNA Transfection Kit (MIR2225; Mirus Bio LLC., Madison, WI, USA), according to the manufacturer's protocol.

\subsection{Quantitation of HEV RNA}

RNA was purified using TRIzol-LS Reagent (Thermo Fisher Scientific) from the culture supernatant, according to the manufacturer's protocol. HEV RNA was then quantitated by quantitative reverse transcription polymerase chain reaction (RT-qPCR) with a 7900HT Fast Real-Time PCR System (Applied Biosystems, Foster City, CA, USA) using specific primers, a TaqMan probe set targeting the ORF2 and ORF3 overlapping region [28], and a QuantiTect Probe RT-PCR Kit (Qiagen, Hilden, Germany), according to the manufacturer's protocol.

\subsection{The Pull-Down Assay}

The FLAG-tagged HEV-like particles were treated with $0.5 \%$ DOC for $1 \mathrm{~h}$ at $37^{\circ} \mathrm{C}$ to remove the envelope. The HEV-like particles were then pelleted and resuspended in Tris-EDTA (TE) buffer (pH 7.6), supplemented with $150 \mathrm{mM} \mathrm{NaCl}$ (TEN). Anti-FLAG M2 magnetic beads were added to the HEV virion suspension, and then incubated for $2 \mathrm{~h}$ at $4{ }^{\circ} \mathrm{C}$. The magnetic beads were rinsed in TEN and treated with $0.5 \%$ DOC only, $0.5 \%$ trypsin only, $0.5 \%$ DOC and $0.5 \%$ trypsin, or $100 \mathrm{mM}$ glycine- $\mathrm{Cl}(\mathrm{pH} 3.5)$. The genomic RNA of the eluted HEV-like particles was quantified by RT-qPCR.

\subsection{Immunofluorescence}

The PLC/PRF/ 5 cells, transfected with the ORF2 (full-length), or the FLAG-tagged ORF2 deletion mutant expression plasmids, were rinsed with $\mathrm{PBS}$ and fixed with $4 \%$ paraformaldehyde/PBS (paraformaldehyde: TAAB Laboratory Equipment Ltd., Reading, UK). The cells were then permeabilized with $0.2 \%$ NP-40/PBS (NP-40, N-6507; Sigma-Aldrich, St. Louis, MO, USA)) and rinsed with PBS. After that, the cells were blocked with $2 \% \mathrm{BSA} / \mathrm{PBS}$ (BSA, A4503; SIGMA) and probed with H6225 or H6210 Mabs, followed by Alexa-488conjugated secondary antibody.

\subsection{HEV Virion Structure Prediction}

The $\mathrm{C} \alpha$ atoms of the previously reported ORF2 structure (3IYO) are displayed as spheres, with a radius of $3.5 \AA$, using the UCSF ChimeraX software program, ver. 1.1 (https://www.rbvi.ucsf.edu/chimerax/; accessed on 6 December 2021). The S (shell, 118-313), M (middle, 314-453), and P (protruding, 455-end) domains are highlighted in blue, green, and orange, respectively.

The human and rat HEV ORF2 protein structures were predicted on the basis of their full-length amino acid sequences, using the AlphaFold2 software program [48]. The predicted human HEV ORF2 structure was superimposed with the previously reported ORF2 structure, 3HAG (genotype 4) [16], 2ZTN (genotype 3) [18], and 6LAT (genotype 1) [27].

In addition, the predicted human HEV ORF2 structure was superimposed on the corresponding region of the chains $\mathrm{A}, \mathrm{B}$, and $\mathrm{D}$ in the $T=3$ icosahedral VLPs (PDB ID: 3IYO) [17], using the UCSF Chimera software program, ver. 1.15 (https: / www.cgl.ucsf. edu/chimera/; accessed on 6 December 2021). The V470-A606 of 3IYO was replaced by 
the V470-S660 of the superimposed human HEV ORF2. The C $\alpha$ atoms of the D118-S469 of $3 \mathrm{IYO}$ are displayed as spheres, with a radius of $3.5 \AA$, using the UCSF ChimeraX software program, ver. 1.1. The amino acid residues, V470-S660, of the superimposed human HEV ORF2 are displayed as surface representations, using the UCSF ChimeraX software program, ver. 1.1. G551-R578 and V579-S660 are highlighted in green and blue, respectively.

Supplementary Materials: The following are available online at https:/ /www.mdpi.com/article/ 10.3390/pathogens11010024/s1, Figure S1: The growth curve of EGFP-tagged HEV, Figure S2: The epitope mapping of H6210, the anti-HEV ORF2 mouse monoclonal antibody, Table S1: The amino acid sequences around R578 of ORF2 proteins among the various HEV strains of genotypes 1-8, Table S2: The amino acid sequences around K567 of rat ORF2 proteins among the various rat HEV strains, Table S3: Primers used in this study.

Author Contributions: Conceptualization, T.N. and H.O.; data curation, T.N. and H.O.; formal analysis, T.N.; funding acquisition, H.O.; investigation, T.N. and K.Y.; methodology, T.N.; project administration, T.N. and H.O.; resources, M.T., S.K. and M.; supervision, K.U. and I.K.; validation, T.N. and H.O.; visualization, T.N.; writing-original draft, T.N. and H.O. All authors have read and agreed to the published version of the manuscript.

Funding: This work was supported, in part, by the Research Program on Hepatitis from the Japan Agency for Medical Research and Development, AMED (JP19fk0210043, JP21fk0210075).

Institutional Review Board Statement: The study was conducted in accordance with the guidelines of the Declaration of Helsinki and was approved by the Institutional Ethics Committee of Jichi Medical University School of Medicine, Tochigi, Japan, under code eki14-82 (21 January 2015). The animal experiments were reviewed and approved by the institutional review board and were performed according to the Guidelines for Animal Experiments at Jichi Medical University, Tochigi, Japan, under code 16-199 (29 March 2016).

Informed Consent Statement: Informed consent was obtained from human subjects involved in the study, as declared in our previous study [49].

Data Availability Statement: Data and methods used in the research are presented in detail in this article. All data relevant to the study are included in the article.

Acknowledgments: We would like to thank the native English-speaking scientists of Japan Medical Communication for the expert linguistic services provided.

Conflicts of Interest: The authors declare no conflict of interest.

\section{Abbreviations}

HEV: hepatitis E virus; Mab: monoclonal antibody; DOC: sodium deoxycholate; VLP: virus-like particle; ORF2tr: truncated ORF2; aa: amino acids; CRE: cis-reactive element.

\section{References}

1. Li, P.; Liu, J.; Li, Y.; Su, J.; Ma, Z.; Bramer, W.M.; Cao, W.; de Man, R.A.; Peppelenbosch, M.P.; Pan, Q. The global epidemiology of hepatitis $E$ virus infection: A systematic review and meta-analysis. Liver Int. 2020, 40, 1516-1528. [CrossRef] [PubMed]

2. Horvatits, T.; Schulze Zur Wiesch, J.; Lütgehetmann, M.; Lohse, A.W.; Pischke, S. The Clinical Perspective on Hepatitis E. Viruses 2019, 11, 617. [CrossRef] [PubMed]

3. Emerson, S.U.; Purcell, R.H. Hepatitis E Virus. Rev. Med. Virol. 2003, 13, 145-154. [CrossRef] [PubMed]

4. Meng, X.-J. Zoonotic and foodborne transmission of hepatitis E virus. Semin. Liver Dis. 2013, 33, 41-49. [CrossRef]

5. Kamar, N.; Selves, J.; Mansuy, J.-M.; Ouezzani, L.; Péron, J.-M.; Guitard, J.; Cointault, O.; Esposito, L.; Abravanel, F.; Danjoux, M.; et al. Hepatitis E virus and chronic hepatitis in organ-transplant recipients. N. Engl. J. Med. 2008, 358, 811-817. [CrossRef]

6. Tavitian, S.; Peron, J.-M.; Huguet, F.; Kamar, N.; Abravanel, F.; Beyne-Rauzy, O.; Oberic, L.; Faguer, S.; Alric, L.; Roussel, M.; et al. Ribavirin for Chronic Hepatitis Prevention among Patients with Hematologic Malignancies. Emerg. Infect. Dis. 2015, 21, 1466-1469. [CrossRef]

7. Shrestha, A.; Adhikari, A.; Bhattarai, M.; Rauniyar, R.; Debes, J.D.; Boonstra, A.; Lama, T.K.; Al Mahtab, M.; Butt, A.S.; Akbar, S.M.F.; et al. Prevalence and risk of hepatitis E virus infection in the HIV population of Nepal. Virol. J. 2017, 14, 228. [CrossRef] 
8. Purdy, M.A.; Harrison, T.J.; Jameel, S.; Meng, X.-J.; Okamoto, H.; Van der Poel, W.H.M.; Smith, D.B. Ictv Report Consortium ICTV Virus Taxonomy Profile: Hepeviridae. J. Gen. Virol. 2017, 98, 2645-2646. [CrossRef]

9. Wang, B.; Meng, X.-J. Hepatitis E virus: Host tropism and zoonotic infection. Curr. Opin. Microbiol. 2021, 59, 8-15. [CrossRef]

10. Li, T.-C.; Bai, H.; Yoshizaki, S.; Ami, Y.; Suzaki, Y.; Doan, Y.H.; Takahashi, K.; Mishiro, S.; Takeda, N.; Wakita, T. Genotype 5 Hepatitis E Virus Produced by a Reverse Genetics System Has the Potential for Zoonotic Infection. Hepatol. Commun. 2019, 3, 160-172. [CrossRef]

11. Wang, L.; Teng, J.L.L.; Lau, S.K.P.; Sridhar, S.; Fu, H.; Gong, W.; Li, M.; Xu, Q.; He, Y.; Zhuang, H.; et al. Transmission of a Novel Genotype of Hepatitis E Virus from Bactrian Camels to Cynomolgus Macaques. J. Virol. 2019, 93, e02014-18. [CrossRef] [PubMed]

12. Sridhar, S.; Yip, C.C.Y.; Wu, S.; Cai, J.; Zhang, A.J.-X.; Leung, K.-H.; Chung, T.W.H.; Chan, J.F.W.; Chan, W.-M.; Teng, J.L.L.; et al. Rat Hepatitis E Virus as Cause of Persistent Hepatitis after Liver Transplant. Emerg. Infect. Dis. 2018, 24, 2241-2250. [CrossRef] [PubMed]

13. Andonov, A.; Robbins, M.; Borlang, J.; Cao, J.; Hatchette, T.; Stueck, A.; Deschambault, Y.; Murnaghan, K.; Varga, J.; Johnston, L. Rat Hepatitis E Virus Linked to Severe Acute Hepatitis in an Immunocompetent Patient. J. Infect. Dis. 2019, 220, 951-955. [CrossRef] [PubMed]

14. Holla, R.P.; Ahmad, I.; Ahmad, Z.; Jameel, S. Molecular virology of hepatitis E virus. Semin. Liver Dis. 2013, 33, 3-14. [CrossRef] [PubMed]

15. Tam, A.W.; Smith, M.M.; Guerra, M.E.; Huang, C.C.; Bradley, D.W.; Fry, K.E.; Reyes, G.R. Hepatitis E virus (HEV): Molecular cloning and sequencing of the full-length viral genome. Virology 1991, 185, 120-131. [CrossRef]

16. Guu, T.S.Y.; Liu, Z.; Ye, Q.; Mata, D.A.; Li, K.; Yin, C.; Zhang, J.; Tao, Y.J. Structure of the hepatitis E virus-like particle suggests mechanisms for virus assembly and receptor binding. Proc. Natl. Acad. Sci. USA 2009, 106, 12992-12997. [CrossRef] [PubMed]

17. Xing, L.; Li, T.-C.; Mayazaki, N.; Simon, M.N.; Wall, J.S.; Moore, M.; Wang, C.-Y.; Takeda, N.; Wakita, T.; Miyamura, T.; et al. Structure of hepatitis E virion-sized particle reveals an RNA-dependent viral assembly pathway. J. Biol. Chem. 2010, 285, 33175-33183. [CrossRef]

18. Yamashita, T.; Mori, Y.; Miyazaki, N.; Cheng, R.H.; Yoshimura, M.; Unno, H.; Shima, R.; Moriishi, K.; Tsukihara, T.; Li, T.C.; et al. Biological and immunological characteristics of hepatitis E virus-like particles based on the crystal structure. Proc. Natl. Acad. Sci. USA 2009, 106, 12986-12991. [CrossRef]

19. Li, S.; Tang, X.; Seetharaman, J.; Yang, C.; Gu, Y.; Zhang, J.; Du, H.; Shih, J.W.K.; Hew, C.-L.; Sivaraman, J.; et al. Dimerization of hepatitis E virus capsid protein E2s domain is essential for virus-host interaction. PLoS Pathog. 2009, 5, e1000537. [CrossRef]

20. Montpellier, C.; Wychowski, C.; Sayed, I.M.; Meunier, J.-C.; Saliou, J.-M.; Ankavay, M.; Bull, A.; Pillez, A.; Abravanel, F.; Helle, F.; et al. Hepatitis E Virus Lifecycle and Identification of 3 Forms of the ORF2 Capsid Protein. Gastroenterology 2018, 154, 211-223.e8. [CrossRef]

21. Okamoto, H. Culture systems for hepatitis E virus. J. Gastroenterol. 2013, 48, 147-158. [CrossRef] [PubMed]

22. Graff, J.; Torian, U.; Nguyen, H.; Emerson, S.U. A bicistronic subgenomic mRNA encodes both the ORF2 and ORF3 proteins of hepatitis E virus. J. Virol. 2006, 80, 5919-5926. [CrossRef] [PubMed]

23. Nagashima, S.; Takahashi, M.; Kobayashi, T.; Tanggis; Nishizawa, T.; Nishiyama, T.; Primadharsini, P.P.; Okamoto, H. Characterization of the Quasi-Enveloped Hepatitis E Virus Particles Released by the Cellular Exosomal Pathway. J. Virol. 2017, 91, e00822-17. [CrossRef] [PubMed]

24. Yin, X.; Ambardekar, C.; Lu, Y.; Feng, Z. Distinct Entry Mechanisms for Nonenveloped and Quasi-Enveloped Hepatitis E Viruses. J. Virol. 2016, 90, 4232-4242. [CrossRef]

25. Takahashi, M.; Tanaka, T.; Takahashi, H.; Hoshino, Y.; Nagashima, S.; Jirintai; Mizuo, H.; Yazaki, Y.; Takagi, T.; Azuma, M.; et al. Hepatitis E Virus (HEV) strains in serum samples can replicate efficiently in cultured cells despite the coexistence of HEV antibodies: Characterization of HEV virions in blood circulation. J. Clin. Microbiol. 2010, 48, 1112-1125. [CrossRef]

26. Yin, X.; Ying, D.; Lhomme, S.; Tang, Z.; Walker, C.M.; Xia, N.; Zheng, Z.; Feng, Z. Origin, antigenicity, and function of a secreted form of ORF2 in hepatitis E virus infection. Proc. Natl. Acad. Sci. USA 2018, 115, 4773-4778. [CrossRef]

27. Zheng, Q.; Jiang, J.; He, M.; Zheng, Z.; Yu, H.; Li, T.; Xue, W.; Tang, Z.; Ying, D.; Li, Z.; et al. Viral neutralization by antibodyimposed physical disruption. Proc. Natl. Acad. Sci. USA 2019, 116, 26933-26940. [CrossRef]

28. Takahashi, M.; Hoshino, Y.; Tanaka, T.; Takahashi, H.; Nishizawa, T.; Okamoto, H. Production of monoclonal antibodies against hepatitis E virus capsid protein and evaluation of their neutralizing activity in a cell culture system. Arch. Virol. 2008, 153, 657-666. [CrossRef]

29. Graff, J.; Nguyen, H.; Kasorndorkbua, C.; Halbur, P.G.; St Claire, M.; Purcell, R.H.; Emerson, S.U. In vitro and in vivo mutational analysis of the $3^{\prime}$-terminal regions of hepatitis e virus genomes and replicons. J. Virol. 2005, 79, 1017-1026. [CrossRef]

30. Wang, B.; Meng, X.-J. Structural and molecular biology of hepatitis E virus. Comput. Struct. Biotechnol. J. 2021, 19, 1907-1916. [CrossRef]

31. Ju, X.; Xiang, G.; Gong, M.; Yang, R.; Qin, J.; Li, Y.; Nan, Y.; Yang, Y.; Zhang, Q.C.; Ding, Q. Identification of functional cis-acting RNA elements in the hepatitis E virus genome required for viral replication. PLoS Pathog. 2020, 16, e1008488. [CrossRef]

32. Olsen, J.V.; Ong, S.-E.; Mann, M. Trypsin cleaves exclusively C-terminal to arginine and lysine residues. Mol. Cell. Proteom. 2004, 3, 608-614. [CrossRef] [PubMed]

33. Cotten, S.W. Evaluation of exocrine pancreatic function. In Contemporary Practice in Clinical Chemistry; Elsevier: Amsterdam, The Netherlands, 2020; pp. 573-585. 
34. Faktor, J.; Goodlett, D.R.; Dapic, I. Trends in Sample Preparation for Proteome Analysis. In Mass Spectrometry in Life Sciences and Clinical Laboratory; IntechOpen: London, UK, 2021.

35. Wei, W.; Behloul, N.; Baha, S.; Liu, Z.; Aslam, M.S.; Meng, J. Dimerization: A structural feature for the protection of hepatitis E virus capsid protein against trypsinization. Sci. Rep. 2018, 8, 1738. [CrossRef] [PubMed]

36. Tiwari, S.P.; Fuglebakk, E.; Hollup, S.M.; Skjærven, L.; Cragnolini, T.; Grindhaug, S.H.; Tekle, K.M.; Reuter, N. WEBnm@ v2.0: Web server and services for comparing protein flexibility. BMC Bioinform. 2014, 15, 427. [CrossRef] [PubMed]

37. Arias, C.F.; DuBois, R.M. The Astrovirus Capsid: A Review. Viruses 2017, 9, 15. [CrossRef]

38. Schofield, D.J.; Glamann, J.; Emerson, S.U.; Purcell, R.H. Identification by phage display and characterization of two neutralizing chimpanzee monoclonal antibodies to the hepatitis E virus capsid protein. J. Virol. 2000, 74, 5548-5555. [CrossRef]

39. Shiota, T.; Li, T.-C.; Yoshizaki, S.; Kato, T.; Wakita, T.; Ishii, K. The hepatitis E virus capsid C-terminal region is essential for the viral life cycle: Implication for viral genome encapsidation and particle stabilization. J. Virol. 2013, 87, 6031-6036. [CrossRef]

40. Kalia, M.; Chandra, V.; Rahman, S.A.; Sehgal, D.; Jameel, S. Heparan sulfate proteoglycans are required for cellular binding of the hepatitis E virus ORF2 capsid protein and for viral infection. J. Virol. 2009, 83, 12714-12724. [CrossRef]

41. Tanaka, T.; Takahashi, M.; Kusano, E.; Okamoto, H. Development and evaluation of an efficient cell-culture system for Hepatitis E virus. J. Gen. Virol. 2007, 88, 903-911. [CrossRef]

42. Jirintai, S.; Mulyanto, T.; Suparyatmo, J.B.; Takahashi, M.; Kobayashi, T.; Nagashima, S.; Nishizawa, T.; Okamoto, H. Rat hepatitis E virus derived from wild rats (Rattus rattus) propagates efficiently in human hepatoma cell lines. Virus Res. 2014, 185, 92-102. [CrossRef]

43. Kobayashi, T.; Takahashi, M.; Mulyanto, T.; Jirintai, S.; Nagashima, S.; Nishizawa, T.; Okamoto, H. Characterization and epitope mapping of monoclonal antibodies raised against rat hepatitis $\mathrm{E}$ virus capsid protein: An evaluation of their neutralizing activity in a cell culture system. J. Virol. Methods 2016, 233, 78-88. [CrossRef] [PubMed]

44. Nishiyama, T.; Kobayashi, T.; Jirintai, S.; Kii, I.; Nagashima, S.; Primadharsini, P.; Nishizawa, T.; Okamoto, H. Screening of novel drugs for inhibiting hepatitis E virus replication. J. Virol. Methods 2019, 270, 1-11. [CrossRef] [PubMed]

45. Lorenzo, F.R.; Tanaka, T.; Takahashi, H.; Ichiyama, K.; Hoshino, Y.; Yamada, K.; Inoue, J.; Takahashi, M.; Okamoto, H. Mutational events during the primary propagation and consecutive passages of hepatitis E virus strain JE03-1760F in cell culture. Virus Res. 2008, 137, 86-96. [CrossRef] [PubMed]

46. Anderson, C.W.; Baum, P.R.; Gesteland, R.F. Processing of adenovirus 2-induced proteins. J. Virol. 1973, 12, 241-252. [CrossRef] [PubMed]

47. Nagashima, S.; Kobayashi, T.; Tanaka, T.; Tanggis; Jirintai, S.; Takahashi, M.; Nishizawa, T.; Okamoto, H. Analysis of adaptive mutations selected during the consecutive passages of hepatitis E virus produced from an infectious cDNA clone. Virus Res. 2016, 223, 170-180. [CrossRef] [PubMed]

48. Jumper, J.; Evans, R.; Pritzel, A.; Green, T.; Figurnov, M.; Ronneberger, O.; Tunyasuvunakool, K.; Bates, R.; Žídek, A.; Potapenko, A.; et al. Highly accurate protein structure prediction with AlphaFold. Nature 2021, 596, 583-589. [CrossRef]

49. Takahashi, M.; Tanaka, T.; Azuma, M.; Kusano, E.; Aikawa, T.; Shibayama, T.; Yazaki, Y.; Mizuo, H.; Inoue, J.; Okamoto, H. Prolonged fecal shedding of hepatitis E virus (HEV) during sporadic acute hepatitis E: Evaluation of infectivity of HEV in fecal specimens in a cell culture system. J. Clin. Microbiol. 2007, 45, 3671-3679. [CrossRef] 